\title{
Improvement of the Critical Speed of the Ball Screw Mechanism by Using Flexible Supported Nut
}

\author{
Songwei Li, Min Wang \\ Beijing Municipal Key Lab of Advanced Manufacturing Technology,Beijing Municipal Key Lab of EDM Technology, College of Mechanical \\ Engineering and Applied Electronics Technology, Beijing University of Technology, Beijing ,100124,China
}

\begin{abstract}
The ball screw with a long stroke has small rigidity in radial direction and is very liable to resonate dramatically during operation at high speed. For improving the critical speed of the ball screw, this paper investigates the influence of the supporting stiffness of the screw shaft at the nut on the resonance frequency of the screw shaft in the radial direction. The screw shaft is modeled as an Euler-Bernoulli beam with elastic supports at both ends and the position of the nut. According to the established model, the relationship between the natural frequencies and the supporting stiffness of the shaft at the nut can be obtained. The simulation results show that the flexible support at the position of the nut can effectively increase the minimum natural frequencies and reduce the variation range of the natural frequencies over the travel range of the nut. It is conducive to the vibration suppression and the critical speed improvement of the ball strew.
\end{abstract}

\section{Introduction}

The ball screw is originated in the late 19th century, which has a high precision, high efficiency, high rigidity, so it is widely used in the mechanical engineering, aviation, aerospace and many other fields [1]. Generally, in the ball screw mechanism, the motor drives the screw shaft to rotate, then the rotary motion of the motor is converted into a liner reciprocation of the nut. The worktable rigidly connected to the screw shaft through the nut, which moves with the travel range of the nut. As an important functional component in high precision machine tools, the performance parameters of the ball strew affect the positioning and repetitive positioning accuracy during the procession, which affect the quality of the workpiece ultimately.

As a linear drive execution unit in the servo feed system of the CNC machine tool, the moving stability and vibration resistance of the ball screw mechanism are important factors affecting the accuracy of the machine tool. Due to the limit of the resonance frequency of the screw shaft, its critical speed is rather difficult to improve. The elongated shaft structure and the large aspect ratio of the ball screw can easily lead to lateral, axial and torsional vibration in the transmission process. During which, the lateral vibration is a common phenomenon. When the variation range of the natural frequencies of the controlled structure is too large, it is difficult to suppress the vibration [2]. In contrast, when the variation range of the natural frequencies of the controlled structure is narrow, the tuned mass dampers or other methods can be used to reduce the vibration, and the effect is also obvious. The mass tuned damper has a simple structure and good stability, which is widely used in the construction, machinery, vehicles and many other industries [3-5].

The nut is continuous moving in the whole stroke, which leads to the alteration of the natural frequencies and mode shapes of the screw shaft, so it is not likely to achieve the vibration control [6]. In terms of the ball screw feed system, scholars have used different methods to build the modeling of the ball screw [7-9], which providing references for the dynamics study of the screw shaft. In previous, most studies did not consider the influence of the travel range of the nut and the supporting stiffness of the screw shaft at the nut on resonance frequency of the screw shaft in the radial direction. This paper mainly studies the lateral vibration of the ball screw, and then builds a dynamic model of the screw shaft, which is elastic supported at both ends and the position of the nut. According to the established model, the relationship between the natural frequencies and the supporting stiffness of the shaft at the nut can be obtained. By optimizing the supporting stiffness of screw shaft at the nut in the radial direction, it is dramatic to increase the minimum natural frequencies and reduce the variation range of the natural frequencies over the travel range of the nut. Based on the optimization results, the flexible supported nut is proposed, which can be used to adjust the supporting stiffness of the screw shaft at the position of the nut and increase the critical rotation speed and vibration resistance of the ball screw. 


\section{The function of the lateral vibration mode shapes of the screw shaft}

In the ball screw feed system, the worktable is connected to the screw shaft with nut, and both ends of the screw shaft are supported by bearings. Among them, there is generally rigid connection between the worktable and the nut.

The screw shaft is modeled as an Euler - Bernoulli beam with elastic supports at both ends and the position of the nut. The length of the screw shaft is $L$, the position of the nut is $L_{1}$, the fulcrum of both ends are $O_{1}$ and $O_{2}$, the origin of coordinates is $O_{1}$, the worktable contacts $O$ point through the nut and the screw shaft, $k_{1}, k_{2}$ represent the radial supporting stiffness of bearings at both ends, $k$ represents the support stiffness of the screw shaft at the nut, the simplified model of the screw shaft is shown in Figure 1.

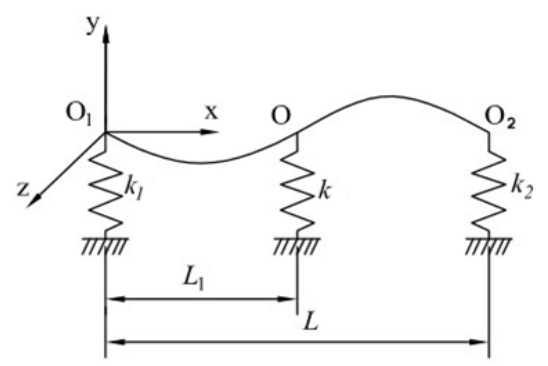

Figure 1. Supporting model of lateral vibration of screw shaft.

According to kinetic equilibrium equations of the beam, the lateral bending vibration mode function is given as follows [10]:

$$
\begin{array}{r}
\varphi(x)=D_{1}[\cosh (\gamma x)+\cos (\gamma x)] \\
+D_{2}[\sinh (\gamma x)+\sin (\gamma x)] \\
+D_{3}[\cosh (\gamma x)-\cos (\gamma x)] \\
+D_{4}[\sinh (\gamma x)-\sin (\gamma x)] \\
A=\pi d^{2} / 4 \\
I=\pi d^{4} / 64 \\
\gamma^{4}=A \omega^{2} \rho /(E I)
\end{array}
$$

where, $D_{1}-D_{4}$ are modal shape modulus, $\omega$ is the natural frequency of the screw shaft, $\rho$ is the density of the screw shaft, $A$ is the cross-sectional area of the screw shaft, $d$ is the diameter of the screw shaft, $E$ is the elastic modulus of the screw shaft, $I$ is the moments of inertia of the screw shaft's cross-section.

Through the formula (1-a) to (1-d), the vibration mode function of $O_{1} O$ segment is expressed as:

$$
\begin{aligned}
\varphi_{1}(x) & =C_{1}[\cosh (\gamma x)+\cos (\gamma x)] \\
& +C_{2}[\sinh (\gamma x)+\sin (\gamma x)] \\
& +C_{3}[\cosh (\gamma x)-\cos (\gamma x)] \\
& +C_{4}[\sinh (\gamma x)-\sin (\gamma x)]
\end{aligned}
$$

The vibration mode function of $\mathrm{OO}_{2}$ segment is written as:

$$
\begin{aligned}
\varphi_{2}(x) & =C_{5}[\cosh (\gamma x)+\cos (\gamma x)] \\
& +C_{6}[\sinh (\gamma x)+\sin (\gamma x)] \\
& +C_{7}[\cosh (\gamma x)-\cos (\gamma x)] \\
& +C_{8}[\sinh (\gamma x)-\sin (\gamma x)]
\end{aligned}
$$

According to the support conditions of the screw shaft at $O_{1}$ and $O_{2}$, the relationship between the bending moment and shear, the following expressions can be obtained.

$$
\begin{aligned}
& \varphi_{1}^{\prime \prime}(0)=0 \\
& E I \varphi_{1}^{\prime \prime \prime}(0)=-k_{1} \varphi_{1}(0) \\
& \varphi_{2}^{\prime \prime}(L)=0 \\
& E I \varphi_{2}^{\prime \prime \prime}(L)=k_{2} \varphi_{2}(L)
\end{aligned}
$$

Considering the screw shaft is continuous at $O$ point, the continuous conditions include displacement, rotation, bending moment and shear, which can be expressed as:

$$
\begin{aligned}
& \varphi_{1}\left(L_{1}\right)=\varphi_{2}\left(L_{1}\right) \\
& \varphi_{1}^{\prime}\left(L_{1}\right)=\varphi_{2}^{\prime}\left(L_{1}\right) \\
& \varphi_{1}^{\prime \prime}\left(L_{1}\right)=\varphi_{2}^{\prime \prime}\left(L_{1}\right) \\
& E I \varphi_{1}^{\prime \prime \prime}\left(L_{1}\right)+k \varphi_{1}\left(L_{1}\right)=E I \varphi_{2}^{\prime \prime \prime}\left(L_{1}\right)
\end{aligned}
$$

By the formula (1) to (11), it is easy to get the screw shaft's natural frequencies $\omega$, modal modulus $C_{1} \sim C_{8}$, and the vibration mode shapes function. In order to make it convenient to calculate, the modal mass of the screw shaft can be normalized, the n-th modal quality of the screw shaft is given as:

$$
m_{n}=\int_{0}^{L_{1}} \rho A \varphi_{n, 1}^{2}(x) d_{x}+\int_{L_{1}}^{L} \rho A \varphi_{n, 2}^{2}(x) d_{x}
$$

The vibration mode function of the screw shaft which is quality normalized can be expressed as follows:

$$
\begin{aligned}
& \varphi_{\mathrm{n}, 1}(x)=\frac{1}{\sqrt{m_{n}}}\left\{\begin{array}{l}
C_{n, 1}\left[\cosh \left(\gamma_{n} x\right)+\cos \left(\gamma_{n} x\right)\right] \\
+C_{n, 2}\left[\sinh \left(\gamma_{n} x\right)+\sin \left(\gamma_{n} x\right)\right] \\
+C_{n, 3}\left[\cosh \left(\gamma_{n} x\right)-\cos \left(\gamma_{n} x\right)\right] \\
+C_{n, 4}\left[\sinh \left(\gamma_{n} x\right)-\sin \left(\gamma_{n} x\right)\right]
\end{array}\right\} \\
& \varphi_{\mathrm{n}, 2}(x)=\frac{1}{\sqrt{m_{n}}}\left\{\begin{array}{r}
C_{n, 5}\left[\cosh \left(\gamma_{n} x\right)+\cos \left(\gamma_{n} x\right)\right] \\
+C_{n, 6}\left[\sinh \left(\gamma_{n} x\right)+\sin \left(\gamma_{n} x\right)\right] \\
+C_{n, 7}\left[\cosh \left(\gamma_{n} x\right)-\cos \left(\gamma_{n} x\right)\right] \\
+C_{n, 8}\left[\sinh \left(\gamma_{n} x\right)-\sin \left(\gamma_{n} x\right)\right]
\end{array}\right\}
\end{aligned}
$$


where, $\varphi_{\mathrm{n}, 1}(x), \varphi_{\mathrm{n}, 2}(x)$ respectively represent the $\mathrm{n}$-th vibration mode function of the screw shaft's $O_{1} O$ and $\mathrm{OO}_{2}$ segment, $\mathrm{C}_{\mathrm{n}, \mathrm{m}}(\mathrm{m}=1,2 \ldots, 8)$ is the modal modulus of the $n$-th vibration mode function.

\section{The analysis of the natural frequencies of the screw shaft}

In the ball screw feed system, the screw shaft is driven by a motor, and then the worktable makes a linear motion accompanied with the screw shaft's rotation. During the whole stroke, the worktable is constantly moving with the travel range of the nut, which lead to the change of the screw shaft's natural frequencies and mode shapes.

In this paper, the screw shaft adopts angular contact ball bearings. The screw shaft's length $L=0.808 \mathrm{~m}$, the screw shaft's diameter $d=0.025 \mathrm{~m}$, the screw shaft's material density $\rho=7.85 \times 10^{3} \mathrm{~kg} / \mathrm{m}^{3}$, and the screw shaft's elastic modulus $E=2.07 \times 10^{11} \mathrm{~Pa}$. According to the calculation formula of the stiffness of angular contact ball bearings, the bearing's supporting stiffness $k_{l}=k_{2}=10^{7} \mathrm{~N}$ $/ \mathrm{m}$.

\subsection{The influence of the travel range of the nut on the natural frequencies of the screw shaft}

Take the supporting stiffness of the screw shaft at the nut in the radial direction $k=10^{7} \mathrm{~N} / \mathrm{m}$. Considering the worktable is moving with the travel range of the nut, the nut's position from $0.1 L$ to $0.9 L$ is selected to calculate. Thus, the natural frequencies over the travel range of the nut are obtained, the results are shown in Figure 2.

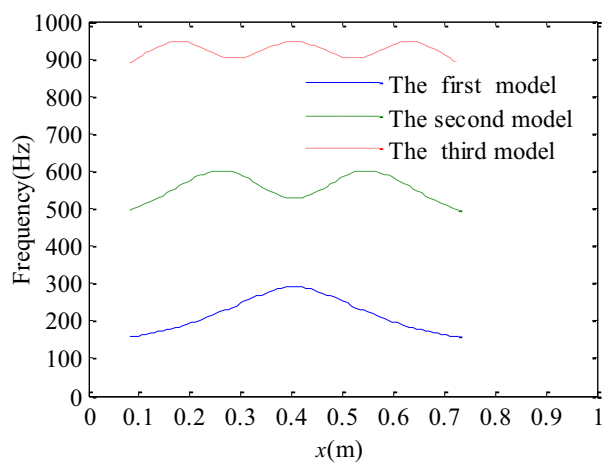

Figure 2. The natural frequencies at the different positions of the nut.

According to Figure 2, During the whole stroke of the nut, the natural frequencies of the screw shaft in radial direction continuously changes within a certain range. With the increase of the vibration models, the variation range of the natural frequencies reduces gradually. The natural frequencies are symmetric with respect to the neutral position over the travel range of the nut. The natural frequency arrives at a peak value in the neutral position. The maximum and minimum values of the second and third natural frequency appear alternately. screw shaft at the nut on the screw shaft's

\section{natural frequencies}

Due to the limitation of screw shaft's natural frequencies, its critical speed is difficult to be improved. It is also necessary to improve the natural frequencies to improve the vibration resistance of the screw shaft. In order to ensure the stability control of the worktable's vibration in the whole stroke, it is necessary to optimize the natural frequencies of the screw shaft. Changing the supporting stiffness of the screw shaft at the nut, the relationship between the natural frequencies and the

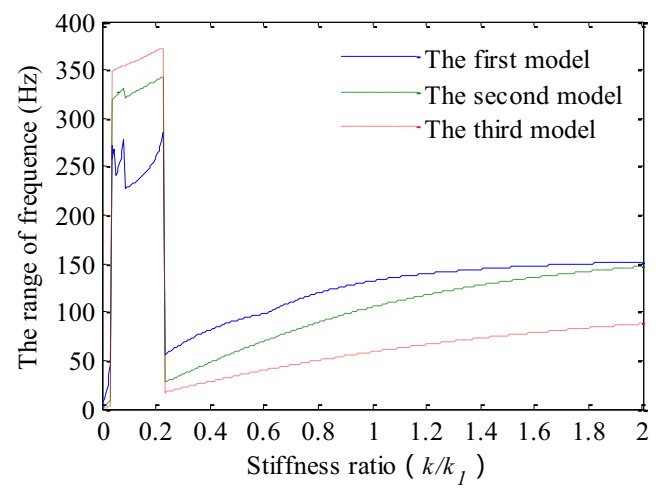

Figure 3. The variation range of the natural frequencies with different supporting stiffness of the screw shaft at the nut

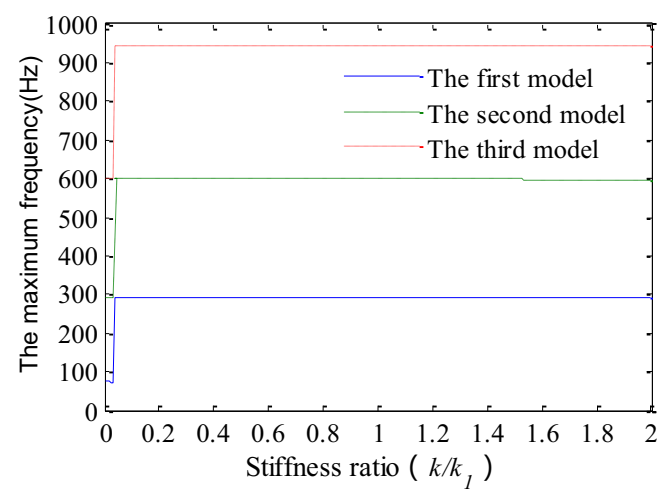

Figure 4. The maximum natural frequencies with different supporting stiffness of the screw shaft at the nut

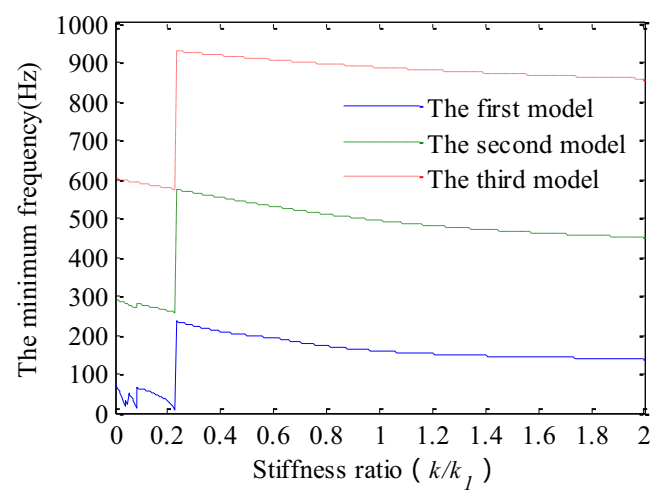

Figure 5. The minimum natural frequencies with different supporting stiffness of the screw shaft at the nut supporting stiffness of the screw shaft at the nut can be obtained.

Considering the travel range of the nut, this paper choose the nut at the positions of $0.1 \mathrm{~L}, 0.2 \mathrm{~L}, 0.3 \mathrm{~L}, 0.4 \mathrm{~L}$ and $0.5 L$ to calculate. Changing the supporting stiffness of the screw shaft at the nut $k$, and the natural frequencies 
at the different positions of the nut can be calculated. Finally, the variation range of the natural frequencies, the minimum and maximum natural frequencies over the travel range of the nut are obtained. Results are shown in Figure 3-Figure 5.

According to Figure 3, during the whole stroke of the nut, the trend of the variation range of the natural frequency is divided into two stages. At the beginning, it increases rapidly with the stiffness ratio varies, which reaches the maximum value ultimately. When the stiffness ratio is about 0.23 , it is reduced to a minimum value quickly. At this time, the natural frequency difference is $53 \mathrm{~Hz}$, the second-order natural frequency difference is $27 \mathrm{~Hz}$, and the third-order natural frequency difference is $18 \mathrm{~Hz}$. When the stiffness ratio is greater than 0.23 , it is gradually reduced, and tends to be stable finally. Figure 4 shows that, the maximum natural frequencies increases rapidly with the stiffness ratio varies, and then it reaches a steady state. Figure 5 shows that, with the increasing of the stiffness ratio, the minimum natural frequencies decreases slowly, and then they reach a peak value suddenly, and finally they decreases to a stable value. When the stiffness ratio is about 0.23 , the minimum natural frequencies arrive at the maximum value.

The results show that optimizing the supporting stiffness of the screw shaft at the nut, the minimum natural frequencies of the shaft over the whole stroke of the nut can be improved obviously, and the variation range of the natural frequencies can be reduced at the same time. According to the optimization results, it is necessary to design a flexible supported nut which can effectively adjust the supporting stiffness of the screw shaft at the position of the nut, and increase the critical rotation speed and vibration resistance of the ball screw mechanism. When designing the flexible supported nut, the stiffness ratio is required to be strict controlled. If the stiffness ratio is relatively too small, the variation range of the natural frequencies is large in the whole stroke, so it is difficult to suppress vibration. Conversely, if the stiffness ratio is relatively too large, the variation range of the natural frequencies is stable, the maximum and the minimum natural frequencies reach a steady state, so there is no use to improve the natural frequencies and suppress vibration with great stiffness ratio. In terms of the screw shaft above, a flexible supported nut whose stiffness ratio is slightly greater than 0.23 can be designed, which can increase the minimum natural frequencies and reduce the variation range of the natural frequencies over the travel range of the nut.

\section{The analysis of the lateral vibration mode shapes of the screw shaft}

Along with the moving of the nut in the range of the whole stroke, the vibration mode shapes of the screw shaft change continuously, which resulting in a negative effect on the running stability of the screw shaft. Mostly, the vibration of the screw shaft occurs in low order natural frequencies, so it is sensible to consider the change of the first vibration mode shape of the screw shaft. Changing the supporting stiffness of the screw shaft at the nut, the vibration mode shape of the screw shaft at the different nut positions is obtained. In order to ensure the modal shape of the screw shaft is stable in the whole stroke of the nut, it is benefit to select the nut at the positions of the $0.1 \mathrm{~L}, 0.3 \mathrm{~L}, 0.5 \mathrm{~L}$ to calculate, the results are shown in Figure 6-8.

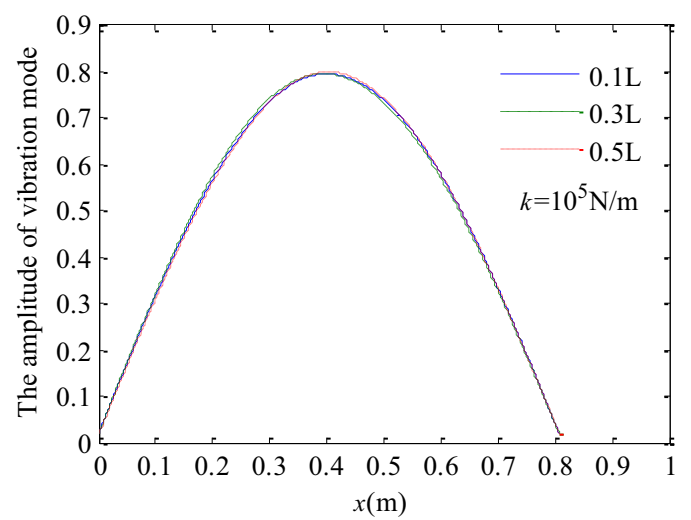

Figure 6. The lateral vibration mode shapes of the screw shaft.

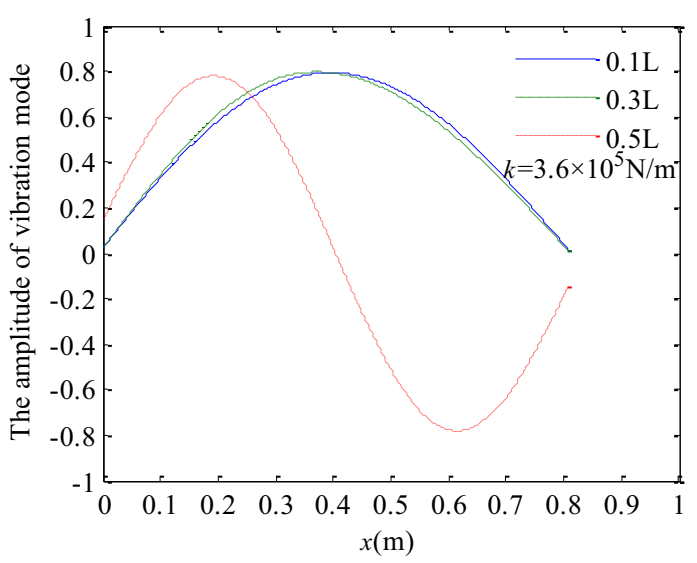

Figure 7. The lateral vibration mode shapes of the screw shaft.

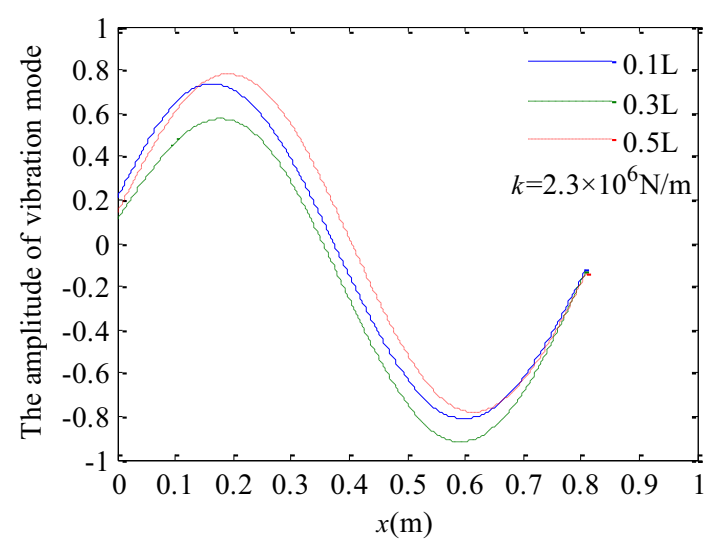

Figure 8. The lateral vibration mode shapes of the screw shaft.

From Figure 6 to Figure 8, when the supporting stiffness of the screw shaft at the nut is changing, the vibration mode shapes is varying. In Figure 6, as the supporting stiffness of the screw shaft at the nut is small, that is to say the screw shaft is similar to the state of no support, so the mode shapes of the screw shaft in the three different nut's position are almost the same. In Figure 7 , when the nut at the position of $0.5 \mathrm{~L}$ and the supporting stiffness of the screw shaft at the nut is 
$3.6 \times 10^{5} \mathrm{~N} / \mathrm{m}$, the vibration mode shape changes suddenly, indicating that the mode shapes of the screw shaft in the whole stroke of the nut is not stable, resulting in the variation range of the natural frequencies change bigger abruptly in Figure 3. When the supporting stiffness of the screw shaft at the nut is $2.3 \times 10^{7} \mathrm{~N} / \mathrm{m}$, the mode shape of the nut at the three different positions are similarity and maintain stable, resulting in the variation range of natural frequencies changes smaller abruptly in Figure 3.

Simulation results show that, when the supporting stiffness of the screw shaft at the nut changes within a certain range, the mode shape of the screw shaft at different nut's positions are varying. The change of the vibration mode shapes of the screw shaft reflect that the natural frequencies of the screw shaft have a big change, resulting in the variation range of natural frequencies change larger in the whole stroke of the nut. By optimizing the supporting stiffness of the screw shaft at the nut, the vibration mode shapes of the screw shaft can remain stable, meanwhile, the variation range of the natural frequencies are the smallest in the whole stroke of the nut. A flexible supported nut can be used to adjust the supporting stiffness of the screw shaft at the nut, it maintains the stability of the vibration mode shapes and reduces the variation range of the natural frequencies of the screw shaft in the whole stroke of the nut effectively, meanwhile, it is also benefit for the smooth operation of the ball screw mechanism.

\section{Conclusions}

By modeling the ball screw mechanism considering the influence of the moving nut to the dynamics of the screw shaft, we analyze the effect of supporting stiffness on the natural frequencies and mode shapes of the screw shaft.
Aiming to control the lateral vibration of the screw shaft, several conclusions can be obtained as follows:

(1) During the whole stroke of the nut, the natural frequencies of the screw shaft in the radial direction continuously changes within a certain range. With the increase of the vibration modes, the variation range of the natural frequencies of the screw shaft in radial direction reduces.

(2) By optimizing the supporting stiffness of the screw shaft at the nut, the minimum natural frequencies of the shaft over the whole stroke of the nut can be improved obviously, and the variation range of the natural frequencies can be reduced at the same time.

(3) According to the optimization results, the flexible supported nut can be used to adjust the supporting stiffness of the shaft at the position of the nut, increase the critical rotation speed and vibration resistance of the ball screw.

\section{References}

1. Z. Xiao, Mach. Tool. Bib , 9 (2001)

2. J. Zhang, Struc. Envi. Engi, 31, 3 (2004)

3. Z. Wang, W.Ren, J. Su, etal. Engi. Mech, 22, 5(2005)

4. Y. Yang, Q. Liu, J. Vibr. Engi, 23, 4 (2010)

5. C. Tong, X. Zhang, J. Vibr. Meas. Diag. 27, 6 (2007)

6. M.Wang, F. Li, T. Zan, X. Gao, J. Vibr. Shock, 34, 10 (2015).

7. H. Zhang, J. Sun, J. Changchun . Univ , 21, 2 (2011)

8. M.C. Lin, B. Ravani,S.A. Velinsky. J. Mech. Design, 116, 3 (1994)

9. G. Holroyd, C. Pislaru, D.G. Ford.Tran. Engi. Sci, 2003

10. D. Zhu, Y. Xing, Engineering Vibration Foundation, (Beihang University Press, 2004) 